ИЗВЕСТИЯ АКАДЕМИИ НАУК ЭСТОНСКОИ ССР. ТОМ 27 ФИЗИКА * МАТЕМАТИКА, 1978, № 4

\title{
ВЛИЯНИЕ ФОНОННОГО ВОЗБУЖДЕНИЯ НА ПЕРЕОРИЕНТАЦИЮ ПРИМЕСНОЙ МОЛЕКУЛЫ В КРИСТАЛЛЕ
}

\author{
(Представлена К. К. Ребане)
}

\section{Введение}

Электронное возбуждение локального центра в кристалле сопровождается, в общем случае, возбуждением фононной подсистемы [ $\left.{ }^{1}\right]$. Последнее носит когерентный характер $\left[{ }^{2}\right]$, который следует учитывать в процессах энергетической и фазовой релаксации возбужденного центра. Ранее было установлено значительное влияние когерентно возбужденного фононного состояния на расплывание колебательной энергии от возбужденного центра $\left[{ }^{2}\right]$, на передачу электронной энергии между центрами $\left[{ }^{3}\right]$, на скорость распада локального колебания в кристаллические $\left[{ }^{4}\right]$, на скорость электронных переходов $\left[{ }^{5}\right]$. В $\left[{ }^{6}\right]$ рассматривалось прямое проявление когерентного колебания в переходных электронно-колебательных спектрах. Представляет интерес рассмотреть еще один тип релаксациөнного процесса с учетом влияния когерентного возбуждения - переориентацию примесной молекулы в кристалле.

\section{Переориентация примесной молекулы}

Гамильтониан вращательно-колебательной подсистемы примесной молекулы в кристалле состоит из вращательного гамильтониана $H_{r}$, колебательного гамильтониана $H_{c}$ и их взаимодействия $H_{r c}$ :

$$
H=H_{r}+H_{c}+H_{r c} \text {. }
$$

В достаточно сильном кристаллическом поле вращательное движение примесной молекулы представляет собой либрации около равновесных орнентаций молекулы и туннельные или фононные переходы между ними. За волновую функцию нулевого приближения берется в этом случае либрационно-колебательная функция в данной ориентации $\psi_{\alpha}$ при условии

$$
E_{\alpha \beta} \equiv\langle H\rangle_{\alpha \beta}=0, \text { если } \alpha \neq \beta
$$

( $\alpha, \beta-$ номера ориентаций). Переориентация происходит за счет возмущения $E_{\alpha \beta} \neq 0$. Вероятность переориентации $\alpha \rightarrow \beta$ вычисляется как элемент $\varrho_{\beta}$ матрицы плотности вращательно-колебательной системы $\varrho(t)=\exp (-i t H / \hbar) \varrho(0) \exp (i t H / \hbar)$ на базисе функций $\psi_{\alpha}$ при начальных условиях

$$
\varrho_{\alpha \alpha}(t=0)=1 ; \quad \varrho_{\beta \gamma}(t=0)=0 ; \quad \beta, \gamma \neq \alpha .
$$


Скорость переориентацин, равная

$$
d_{\mathrm{Q \beta \beta}} / d t \text {, }
$$

определяется в возбужденном электронном состоянии как среднее значение

$$
K=\Gamma^{-1} \int_{0}^{\infty} d t \exp (-\Gamma t)\left[d_{\varrho_{\beta} \beta} / d t\right]
$$

где $\Gamma$ - константа затухания возбужденного электронного состояния.

Скорость переориентации (4) рассчитывалась нами в следующих предположениях:

1. Переориентация является необратимым процессом $\alpha \rightarrow \beta$, а не процессом типа пульсации между $\alpha$ и $\beta$, поскольку из-за неоднородностей кристалла ориентации неэквивалентны, т. е. $E_{\alpha \alpha} \neq E_{\beta \beta}$, причем их разность больше $E_{\alpha \beta}$.

2. Вращательно-колебательное взаимодействие диагональное в $\boldsymbol{\alpha}$-представлении и линейное по нормальным координатам $q_{s}$, т. е.

$$
\left\langle H_{c}+H_{r c}\right\rangle_{\alpha \beta}=\delta_{\alpha \beta} \sum_{s} \frac{1}{2} \pi \omega_{s}\left(q_{s}-q_{s 0}^{\alpha}\right)^{2} .
$$

Таким образом, гармонические потенциалы колебаний разных ориентаций $\alpha$ представляются как многомерные параболоиды, смещенные и по энергии и по координатам.

3. Недиагональное возмущение $E_{\alpha \beta}$ не зависит от колебательных коордннат.

4. Матрица плотности когерентного начального состояния колебаний задается через оператор смещения $[2,4,5]$ как

$$
\mathrm{Q} \alpha \alpha_{\alpha}(0)=P_{\mathrm{Q}_{0}} P^{-1},
$$

где $\varrho_{0}$ - термически равновесный оператор плотности, а оператор смещения имеет вид

$$
P=\exp \left(-\sum_{s} p_{s 0} \frac{d}{d q_{s}}\right)
$$

где $p_{s 0}$ - амплитуда когерентного состояния колебания номера $s$.

В этих предположениях, ограничиваясь при вычислении е первыми членами разложения по степеням $H_{r}$, можем записать

$$
\begin{gathered}
d_{\varrho \beta \beta} / d t=2\left|E_{\alpha \beta}\right|^{2} \bar{t}^{-2} \exp \left\{-\sum_{s}\left(q_{s 0}^{\alpha \beta}\right)^{2}\left(\bar{n}_{s}+\frac{1}{2}\right)\right\} \times \\
\times \operatorname{Re} \int_{0}^{t} d_{\varrho} \exp \left\{i \omega_{\alpha \beta \varrho}+\frac{1}{2} \sum_{s}\left(q_{s 0}^{\alpha \beta}\right)^{2}\left[\bar{n}_{s} \exp \left(i \omega_{s} \varrho\right)+\left(\bar{n}_{s}+1\right) \exp \left(-i \omega_{s} \varrho\right)\right]+\right. \\
+\frac{1}{9} \sum_{s} q_{s 0}^{\alpha \beta} p_{s 0}\left[\exp \left(-i \omega_{s} t\right)-\exp \left(i \omega_{s} t\right)-\exp \left(-i \omega_{s}(t-\varrho)\right)+\right. \\
\left.\left.+\exp \left(i \omega_{s}(t-\varrho)\right)\right]\right\} .
\end{gathered}
$$

Здесь $\bar{n}_{s}$ - среднее квантовое число колебания номера $s$ с частотой $\omega_{s}$, a

$$
q_{s 0}^{\alpha \beta}=q_{s 0}^{\alpha}-q_{s 0}^{\beta} \quad \text { и } \quad \omega_{\alpha \beta}=\left(E_{\alpha \alpha}-E_{\beta \beta}\right) / \hbar .
$$


Первая экспонента в формуле (8) учитывает перенормировку туннельного матричного элемента из-за взаимодействия переорнентации с колебаниями кристалла $\left[{ }^{7,8}\right]$. Этот бесфононный фактор может составлять у примесных молекул в щелочногалоидных кристаллах величину до $10^{-13}\left[{ }^{9}\right]$. Он был учтен в прежних расчетах скорости переориентации для термически равновесного $(P=1)$ начального состояния $\left[{ }^{10,11}\right]$.

Члены $m$-го порядка разложения экспоненты

$$
\exp \left\{\frac{1}{2} \sum_{s}\left(q_{s 0}^{\alpha \beta}\right)^{2}\left[\bar{n}_{s} \exp \left(i \omega_{s} \varrho\right)+\left(\bar{n}_{s}+1\right) \exp \left(-i \omega_{s} \varrho\right)\right]\right\}
$$

в формуле $(8)$ по степеням ехр $\left( \pm i \omega_{s} \varrho\right)$ приводят соответственно к скоростям $m$-фононных переориентаций.

Формула (8) переходит в формулу кинетического коэффициента $\left[{ }^{5}\right]$, если $\omega_{\alpha \beta}$ принять за частоту электронного перехода и предполагать когерентное возбуждение колебаний только фиксированной частоты. Последняя сумма в формуле (8) определяет уже вклад когерентного пакета кристаллических колебаний любой частотной ширины, учитывая тем самым и затухающие когерентные начальные состояния.

Отметим, что индекс состояния $\alpha$ означает не только номер ориентации, но и квантовые числа состояния молекулы. Тем самым формула (8) относится и к переориентациям в возбужденных состояниях либрационных или внутримолекулярных (локальных) колебаний; и к переориентациям с изменением этих состояний или даже с нзменением электронного состояния, если удовлетворен критерий адиабатического приближения для этой пары электронных состояний. В последних случаях к энергии $E_{\alpha \alpha}-E_{\beta \beta}$ прибавляется еще энергия соответствующего квантового перехода.

Имеется аналогия между вибронным переходом и процессом переориентации по данной модели $\left[{ }^{8}\right]$, причем энергии электронного перехода соответствует разность $E_{\beta \beta}-E_{\alpha \alpha}$, а стоксовому смещению - разность $q_{s 0}^{\alpha}-q_{s 0}^{\beta}$.

\section{Сиорость переориентации в возбужденном электронном состоянии}

Скорость переориентации примесной молекулы в возбужденном электронном состоянии определяется формулами (5) и (8). Для выяснения ее основных зависимостей от энергий и констант затухания когерентного состояния можно предположить в формуле (8) для величин $\left(q_{s 0}^{\alpha \beta}\right)^{2}$ и $q_{s 0}^{\alpha \beta} p_{s 0}$ лоренцево частотное распределение полуширины $\gamma$. Такая аппроксимация означает введение эффективного псевдолокального колебания частоты $\bar{\omega}$, когерентно возбужденного в начальный момент и затухающего с константой $\gamma$.

В предположении сильного взаимодействия переориентации с колебаниями (это соответствует случаю больших стоксовых потерь в вибронном переходе)

$$
L=\frac{1}{2} \sum_{s}\left(q_{s 0}^{\alpha \beta}\right)^{2} \gg 1
$$

формулу (8) можно преобразовать в приближенное выражение

$$
d_{Q \beta \beta} / d t=\left|E_{\alpha \beta}\right|^{2 \hbar-2} \sqrt{2 \pi / L \bar{\omega}^{2}} \exp \left\{-(L+2 M \cos \bar{\omega} t)^{2} / 2 L\right\} .
$$


Здесь $M=M_{0} \exp (-\gamma t), M_{0}=\frac{1}{2} \sum_{s} q_{s 0}^{\alpha \beta} p_{s 0}, \quad$ а также предположено, что $\omega_{\alpha \beta} \prec \bar{\omega}$ и $\bar{n}(\bar{\omega}) \approx 0$.
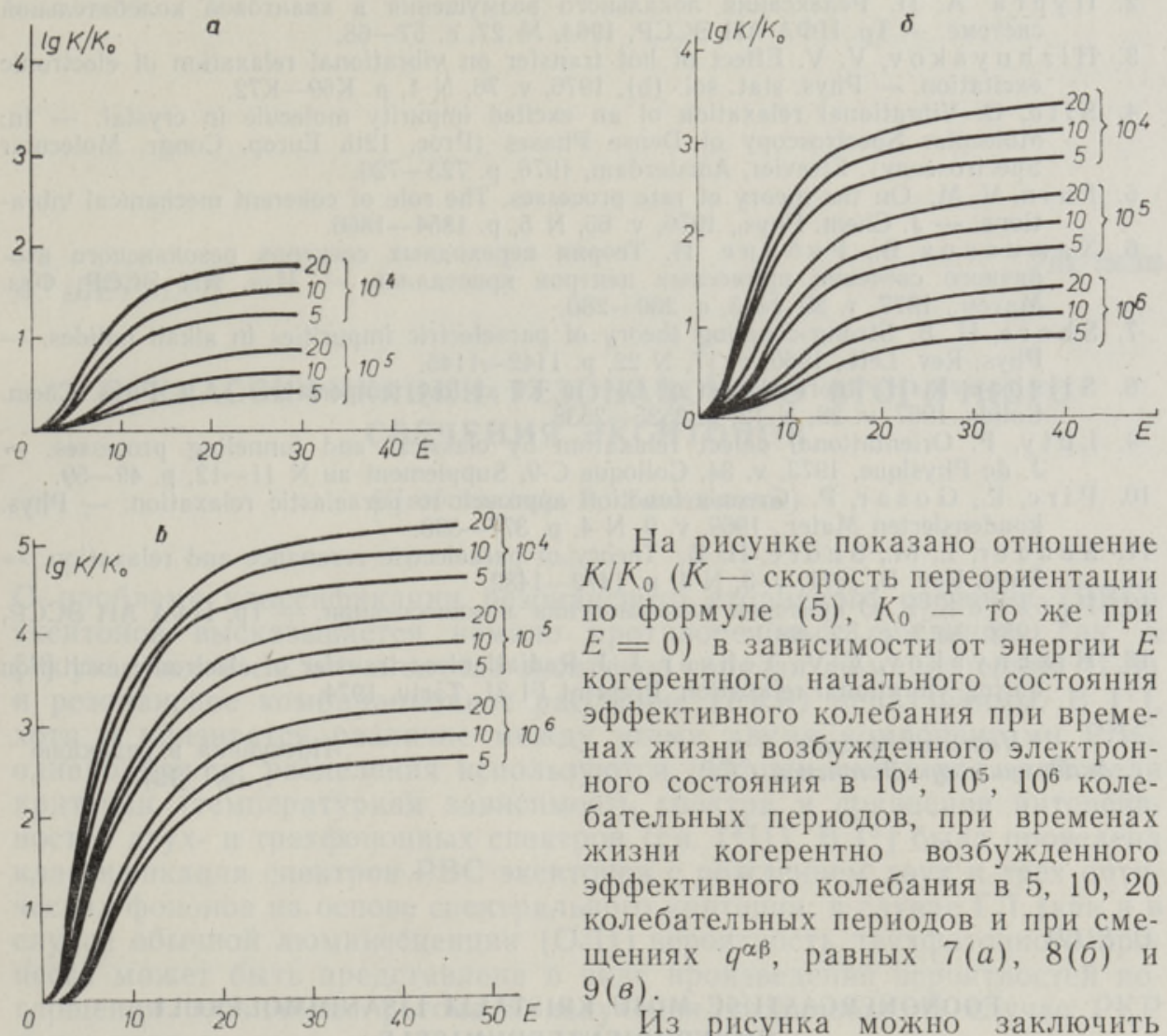

На рисунке показано отношение $K / K_{0}(K-$ скорость переориентации по формуле (5), $K_{0}-$ то же при $E=0$ ) в зависимости от энергии $E$ когерентного начального состояния эффективного колебания при временах жизни возбужденного электронного состояния в $10^{4}, 10^{5}, 10^{6}$ колебательных периодов, при временах жизни когерентно возбужденного эффективного колебания в $5,10,20$ колебательных периодов и при смещениях $q^{\alpha \beta}$, равных $7(a), 8(б)$ и $9(8)$.

Из рисунка можно заключить следующее. Заметное ускорение переориентации, обусловленное когерентным начальным состоянием, имеет место при смещениях $q^{\alpha \beta}$, бо́льших 6 . Это соответствует большим стоксовым потерям при переориентации (больше 20 колебательных квантов). При этом начальная амплитуда когерентного состояния должна достигать примерно половины $q^{\alpha \beta}$. Это означает, что переориентация ускоряется колебательным возбуждением, энергетическая релаксация которого «проходит точку пересечения» сильно смещенных адиабатических потенциалов начальной и конечной ориентаций.

Когерентно возбужденное фононное состояние, в котором ядра около примеси испытывают конечные смещения, является квантовомеханическим состоянием, наиболее близким к классическому состоянию колебания этих ядер. Поэтому рассмотренная выше модель описывает разные процессы перехода между двумя адиабатическими поверхностями в условиях высокоэнергетических (близких к классическим) фононных возбуждений. Ускорение, например, безызлучательного электронно-колебательного перехода с колебательных состояний выше точки пересечения адиабатических потенциалов было установлено в $\left[{ }^{12,13}\right]$.

Фононное возбуждение, ускоряющее релаксационный процесс, может быть результатом не только электронного возбуждения примеси, но и результатом, напр., распада высокочастотного локального колебания. 


\section{Л И Т Е Р А Т У Р А}

1. Р ебане К. Қ. Элементарная теория колебательной структуры спектров примесных центров кристаллов. М., 1968, гл. 1.

2. Пурга А. П. Релаксация локального возмущения в квантовой колебательной системе. - Тр. ИФА АН ЭССР, 1964, № 27, с. 57-68.

3. Hizhnyakov, V. V. Effect of hot transfer on vibrational relaxation of electronic excitation. - Phys. stat. sol. (b), 1976, v. 76, N 1, p. K69-K72.

4. Sild, O. Vibrational relaxation of an excited impurity molecule in crystal. - In: Molecular Spectroscopy of Dense Phases (Proc. 12th Europ. Congr. Molecular Spectroscopy). Elsevier, Amsterdam, 1976, p. 723-726.

5. F a in, V. M. On the theory of rate processes. The role of coherent mechanical vibrations. - J. Chem. Phys., 1976, v. 65 , N 5, p. $1854-1866$.

6. Хи жняков В., Р еб ан е И. Теория переходных спектров резонансного вторичного свечения примесных центров кристаллов. - Изв. АН ЭССР, Физ. Матем., 1977 , т. 26 , № 3, с. $260-280$.

7. Shore, H. B. Strong-coupling theory of paraelectric impurities in alkali halides. Phys. Rev. Lett., 1966, v. 17, N 22, p. 1142-1145.

8. $\mathrm{S}$ ils bee, R. H. Reorientation of $\mathrm{O}_{2}{ }^{-}$in $\mathrm{KI}$ at low temperature. - J. Phys. Chem. Solids, 1967 , v. 28 , N 12 , p. $2525-2538$.

9. L üt y, F. Orientational defect relaxation by classical and tunneling processes. J. de Physique, 1973, v. 34, Colloque C-9, Supplement au N 11-12, p. 49-59.

10. P i r c, R., Gos a r, P. Green's function approach to paraelastic relaxation. - Phys. kondensierten Mater., 1969, v. 9, N 4, p. 377-398.

11. S a nder, L. M., Shore, H. B. Theory of paraelectric resonance and relaxation. Phys. Rev. B, 1971 , v. 3 , N 4, p. $1472-1482$.

12. Р е б а н е К. К. О критерии возникновения люмннесценции. - Тр. ИФА АН ЭССР, 1958 , № 7 , c. $62-84$.

13. Hizhny a kov, V. V., Te hver, I. J. Radiationless transfer of electronic excitation during vibrational relaxation. Preprint FI-31, Tartu, 1974.
Ннститут физики
Академии наук Эстонской ССР
Поступила в редакцию
$2 / \mathrm{I} \quad 1978$

\section{FOONONERGASTUSE MOJU KRISTALLI LISANDIMOLEKULI OMBERORIENTEERUMISELE}

Artiklis on käsitletud kristalli lisandimolekuli ümberorienteerumist koherentse foononergastuse korral ning arvutatud selle kiiruse sõltuvus foononergastuse energiast ja elueast.

\section{O. SILD}

\section{THE REORIENTATION OF THE IMPURITY MOLECULE IN CRYSTAL AT PHONON EXCITATION}

The reorientation of the impurity molecule in crystal at coherent phonon excitation is examined. The reorientation rate for excited electronic state is calculated (formulae (5) and (8)). The dependence of the reorientation rate on the optical lifetime, on the energy and lifetime of the phonon excitation as well as on the shift $q$ of the initial and final orientation adiabatic potentials is demonstrated.

The reorientation rate increases in the coherent phonon excitation. The figure represents the relation $K / K_{0}\left(K-\right.$ reorientation rate after formula $(5)$ and $K_{0}$ - reorientation rate at $E=0$ ) as the function of the phonon excitation energy $E$, for the optical lifetimes of $10^{4}, 10^{5}$ or $10^{6}$ vibration periods and for the phonon excitation lifetimes of 5,10 or 20 vibration periods. The figure parts $a, \sigma, 8$ are calculated for the shifts $q=7, q=8$ and $q=9$, respectively. An essential enhancement of the reorientation rate takes place at $q>6$ in case the initial phonon excitation energy $E$ exceeds the crossing point of the adiabatic vibration potentials of the initial and final orientations. 